\title{
Tc-99m HMDP versus MDP discrepancy in cardiac amyloidosis
}

An important point was highlighted in the conclusion of Cuscaden et al. ${ }^{1}$ We want to draw attention to this point because there is some misconception regarding this issue in nuclear amyloid imaging. Uptake of hydroxymethylene diphosphonate (HMDP) is intense in myocardium infiltrated with transthyretin amyloidosis. Uptake of methylene diphosphonate (MDP), on the other hand, may not be intense at all.

These two employ the same mechanism in bone scans. These radiolabelled bisphosphonates bind to hydroxyapatite at sites of active bone formation (osteogenesis). Osteogenesis is a nonspecific response of bone to a range of stimuli, such as physiological growth/turnover, mechanical stress or injury (including fractures), infection and involvement by tumor ${ }^{2}$.

They have, however, very discrepant characteristics with amyloid imaging. This discordance was underlined by Cuscaden et al. ${ }^{1}$ by the significantly different incidence of transthyretin cardiac amyloidosis detected by the two radiotracers. This clearly showed that HMDP and MDP are not interchangeable in the context of amyloid imaging. Current guidelines ${ }^{3}$ address the use of Tc99m PYP/DPD/HMDP and, appropriately, do not mention MDP. Nevertheless, the use of Tc99m MDP for this purpose may be applied by some nuclear medicine physicians.

To avoid this misapprehension, perhaps it ought to be stated explicitly in relevant guidelines. The current consensus of the major relevant societies discusses the methodology and interpretation with reference only to "bone-avid radiotracers", We call on future guidelines to formally curb this misconception of interchangeability.

\author{
Joseph C. Lee, MBBS, FRACP, FAANMS, ${ }^{a, b}$ \\ Nathan Better, MBBS, FRACP, FAANMS, FCSANZ, \\ FASNC $C^{c, d}$ \\ ${ }^{a}$ Department of Medical Imaging, The Prince Charles Hospital, \\ Chermside, QLD 4032, Australia \\ ${ }^{b}$ Faculty of Medicine, University of Queensland, Herston, Australia \\ joseph.lee@health.qld.gov.au \\ ${ }^{c}$ Department of Nuclear Medicine, Royal Melbourne Hospital, Grattan \\ St, Melbourne, VIC 3000, Australia \\ ${ }^{d}$ Department of Medicine, University of Melbourne, Parkville, \\ Australia
}

\section{Author Contributions}

Conception or design of the work; or the acquisition, analysis, or interpretation of data for the work: JCL 60\% NB $40 \%$. Drafting the work or revising it critically for important intellectual content: JCL $60 \%, N B 40 \%$

\section{Disclosures}

Dr Lee has received travel expenses for attending meetings of his specialty College's Continuing Professional Development Committee, of which he is a member.

\section{References}

1. Cuscaden C, Ramsay SC, Prasad S, Goodwin B, Smith J. Estimation of prevalence of transthyretin (ATTR) cardiac amyloidosis in an Australian subpopulation using bone scans with echocardiography and clinical correlation. J Nucl Cardiol. 2020. https://doi.org/10.1007/s12350-020-02152-x

2. Lee JC, Hennessy AD, Khafagi FA. Bone scans. Aust Fam Phys 2012;41:689-92

3. Dorbala S, Ando Y, Bokhari S, Dispenzieri A, Falk RH, Ferrari VA, Fontana $\mathrm{M}$ et al. ASNC/AHA/ASE/EANM/HFSA/ISA/SCMR/ SNMMI expert consensus recommendations for multimodality imaging in cardiac amyloidosis: Part 1 of 2-evidence base and standardized methods of imaging. J Nucl Cardiol 2019;26:2065-23 doi:10.1007/s12350-021-02763-y

Publisher's Note Springer Nature remains neutral with regard to jurisdictional claims in published maps and institutional affiliations.

J Nucl Cardiol 2021;28:2424.

$1071-3581 / \$ 34.00$

Copyright (c) 2021 American Society of Nuclear Cardiology. 